\title{
Modulating the Conductance in Graphene Nanoribbons with Multi-Barriers Under an Applied Voltage
}

\author{
Hassen Dakhlaoui, ${ }^{1,2}$ Shaffa Almansour, ${ }^{1,2}$ Walid Belhadj, ${ }^{3}$ and Bryan M. Wong ${ }^{4, *}$ \\ ${ }^{1}$ Nanomaterials Technology unit, Basic and Applied Scientific Research Center (BASRC), College of Science of \\ Dammam, Imam Abdulrahman Bin Faisal University, P. O. Box 1982, 31441 Dammam, Saudi Arabia. \\ ${ }^{2}$ Department of Physics, College of Sciences for Girls, Imam Abdulrahman Bin Faisal University, Saudi Arabia \\ ${ }^{3}$ Department of Physics, College of Applied Sciences, Umm Al-Qura University, Mecca, Saudi Arabia \\ ${ }^{4}$ Department of Chemical \& Environmental Engineering, Materials Science \& Engineering Program, and Department \\ of Physics and Astronomy, University of California-Riverside, Riverside, CA USA \\ *Email: bryan.wong@ucr.edu, Website: http://www.bmwong-group.com
}

\begin{abstract}
The electronic transmission and conductance produced by Dirac electrons in an armchair graphene nanoribbon under an external voltage are investigated with the transfer matrix method. We investigate the velocity and voltage of nanoribbons in the presence of single and multiple barriers and show that the transmission coefficients can be controlled by varying the order of the mode, the number of carbon atoms, and the barrier velocities. In particular, we find that the nanoribbon appears to be fully transparent when the barrier and Fermi velocities are equal. Our numerical results show that the electronic conductance is sensitive to the applied external voltage and number of carbon atoms, which can be used to tailor the electronic properties of graphenebased devices.
\end{abstract}

\section{Introduction}

The electronic and optical properties in graphene-based heterostructures continue to garner immense interest due to their technological applications in new devices such as graphene-based memory, field-effect transistors, and molecule sensors [1-5]. In particular, many of these devices utilize nanoribbons, which exhibit electronic properties that considerably differ from bulk graphene [6-9]. For example, a monolayer graphene sheet is a semimetal with a zero gap between the conduction and valence bands; however, nanoribbon materials have a non-zero bandgap and can be either metallic or semiconducting, depending on their width.

In contrast to single-well quantum structures, superlattices can be harnessed for manipulating the electronic properties of semiconductor materials [10-12]. In particular, graphenebased superlattices that have ordered or disordered superlattices [13-18] can be controlled and manipulated via an applied voltage, magnetic field, and Fermi-velocity barriers [19-27]. For instance, applying a moderate external magnetic field within the barrier regions can break valley degeneracies for use in valleytronic devices [28, 29]. Moreover, magnetic fields can be used to ensure the opening and closing of the energy gap in metallic armchair-edge graphene nanoribbons (AGNs) [30]. 
In addition, the transmission coefficient and electronic conductance can also be modulated by tuning the intensity of an applied voltage or by varying the widths of the quantum wells and barriers of the heterostructure. Modulating the Fermi velocity [31] can also tune the energy gap to adjust the desired transmission from 0 to 1 , which can effectively alter the on/off states in graphenebased transistors. The Fermi velocity in AGNs can be modulated by different techniques such as the insertion of impurities, strain, and applied electric fields [32]. In previous work, Haiyan Wang et al. studied the electronic properties of AGNs with double magnetic barriers and showed that the conductance exhibits quantized plateaus in both metallic and semiconducting cases [33]. The electronic properties of AGNs modulated with a potential barrier were examined by Benliang Zhou et al., [34] who showed that the conductance could exhibit distinct minima when the barrier width was altered. Nascimento et al. [35] studied the electron transport of Dirac electrons through a nanoribbon and showed that the Fermi and barrier velocities could be tuned to modulate the electronic properties of graphene-based materials. Despite the previous studies on this topic, an investigation of the transmission coefficient and electronic conductance through multibarrier armchair graphene nanoribbons under the effect of an applied voltage has not been carried out. To this end, we examine the electronic transmission and conductance under an applied voltage, barrier velocity, and different numbers of barriers and carbon atoms. The present paper is structured as follows: Section 2 outlines our theoretical model, Section 3 presents our numerical results, and finally, Section 4 summarizes our important findings and conclusions.

\section{Theory}

In the present paper, we consider an AGN in the $x y$-plane that is subjected to an applied external voltage. The voltage acts only on the substrates that constitute the barriers. These substrates are also used to adjust the value of the barrier velocity $v_{b}$, which will be expressed as a function of the Fermi velocity $v_{F}$. A schematic of the system under study is presented in Fig. 1.

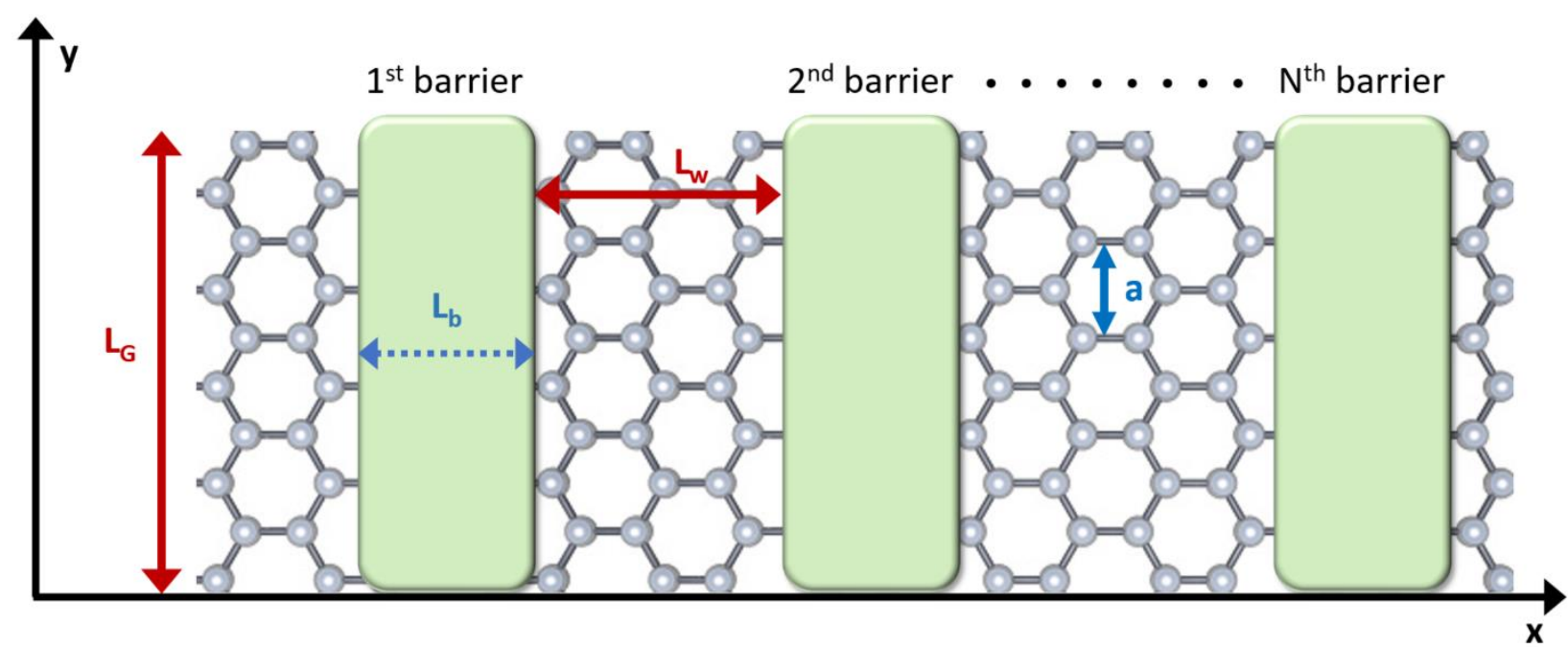

Figure1: Schematic diagram of an armchair graphene nanoribbon. The total width of the nanoribbon is $L_{G} . L_{b}$ and $L_{w}$ denote the barrier and channel widths, respectively. 
The motion of Dirac electrons is described by the following Hamiltonian [31, 32, 35]:

$$
H=-i \hbar\left(\sqrt{v_{F}(x)} \sigma_{x} \partial_{x} \sqrt{v_{F}(x)}+v_{F}(x) \sigma_{y} \partial_{y}\right)+V(x),
$$

where $\hbar$ is the reduced Planck constant, $v_{F}(x)$ is the Fermi velocity, and $\sigma_{x, y}$ are the Pauli matrices. These matrices act on the pseudo-spin wavefunctions describing the two sublattices of the graphene nanoribbons. In this situation, the two spinor wavefunctions associated with the total Hamiltonian are $\Psi(x, y)=\left[\Psi_{A}(x, y), \Psi_{B}(x, y)\right]$, where the $A$ and Bindices denote the two graphene sublattices. By making the change of variable $\Psi(x, y)=\frac{\phi(x, y)}{\sqrt{v_{F}(x)}}$, equation (1) can be readily transformed as [32]:

$$
H \phi(x, y)=\left(-i \hbar v_{F}(x)\left(\sigma_{x} \partial_{x}+\sigma_{y} \partial_{y}\right)+V(x)\right) \phi(x, y)
$$

The motion along $x$ and $y$ can be decoupled, and the wavefunction $\phi(x, y)$ can be written as $\phi(x, y)=\Phi(x) \exp \left(i k_{y} y\right)$. Equation (2) can be transformed to the following [32]:

$$
\left(\begin{array}{cc}
V(x) / \hbar v_{F}(x) & -i\left(\partial_{x}+k_{y}\right) \\
-i\left(\partial_{x}-k_{y}\right) & V(x) / \hbar v_{F}(x)
\end{array}\right)\left(\begin{array}{c}
\Phi_{A} \\
\Phi_{B}
\end{array}\right)=E / \hbar v_{F}(x)\left(\begin{array}{c}
\Phi_{A} \\
\Phi_{B}
\end{array}\right) .
$$

The total length of the nanoribbon is fixed to $L_{G}$; therefore, the wavevector $k_{y}$ satisfies the boundary condition:

$$
k_{y} L_{G}=\pi(m+\beta), \quad m=0, \pm 1, \pm 2, \ldots
$$

In addition, the total length of the nanoribbon $L_{G}$ is related to the number of carbon atoms $p$ by the relation $L_{G}=(p+1) a / 2$, where $a=0.246 \mathrm{~nm}$ [36]. For instance, the schematic in Fig. 1 has $p=$ 8 with a total length of $L_{G}=9 a / 2$.

In equation (4), $m$ labels the mode of propagation, and $\beta$ is equal to $1 / 3(0)$ for a semiconductor (metallic) nanoribbon [37]. In matrix equation (3), the applied potential $V(x)$ and the Fermi velocity $v_{F}(x)$ are given in the barrier and well regions by the following expressions $[35,36]$ :

$$
\begin{gathered}
V(x)=\left\{\begin{array}{l}
0 \text { in quantum well regions } \\
V \text { in barrier regions }
\end{array},\right. \\
v_{F}(x)=\left\{\begin{array}{l}
v_{F} \text { in quantum well regions } \\
v_{b} \text { in barrier regions }
\end{array} .\right.
\end{gathered}
$$

By solving equation (3), the wavefunctions describing the Dirac electrons in the quantum well and barrier regions can be written as: 


$$
\begin{gathered}
\Phi_{\text {well }}=T_{W}\left(\begin{array}{l}
A_{m} \\
B_{m}
\end{array}\right)=\left(\begin{array}{cc}
e^{i k x} & e^{-i k x} \\
\frac{k_{x}+i k_{y}}{E} e^{i k x} & \frac{-k_{x}+i k_{y}}{E} e^{i k x}
\end{array}\right)\left(\begin{array}{l}
A_{m} \\
B_{m}
\end{array}\right), \\
\Phi_{\text {barrier }}=T_{b}\left(\begin{array}{c}
C_{m} \\
D_{m}
\end{array}\right)=\left(\begin{array}{cc}
e^{i q x} & e^{-i q x} \\
\frac{q_{x}+i k_{y}}{E-V} e^{i k x} & \frac{-q_{x}+i k_{y}}{E-V} e^{i k x}
\end{array}\right)\left(\begin{array}{c}
C_{m} \\
D_{m}
\end{array}\right),
\end{gathered}
$$

where the wavevectors $k_{x}$ and $q_{x}$ are given by:

$$
\begin{gathered}
k_{x}=\sqrt{\left(\frac{E}{\hbar v_{F}}\right)^{2}-k_{y}^{2},} \\
q_{x}=\sqrt{\left(E-\frac{V}{\hbar v_{b}}\right)^{2}-k_{y}^{2} .}
\end{gathered}
$$

$T_{W}$ and $T_{b}$ denote the transfer matrix [39] in the well and barrier regions, respectively. Using the continuity of the wavefunction in each barrier interface, the total transfer matrix through the $j+1$ barrier can be obtained as follows:

$$
T_{j}=T_{w}^{-1}\left(x_{j}\right) T_{b}\left(x_{j}\right) T_{b}^{-1}\left(x_{j}+L_{w}\right) T_{w}\left(x_{j}+L_{w}\right) .
$$

For the $N^{\text {th }}$ barrier, the total transfer matrix of the system becomes:

$$
T=\left(\begin{array}{ll}
T_{11} & T_{12} \\
T_{21} & T_{22}
\end{array}\right)=\prod_{i=0}^{N-1} T_{j} .
$$

Finally, the transmission coefficient for the $m^{\text {th }}$ mode is readily given by

$$
T_{m}(E)=\frac{1}{\left|T_{11}\right|^{2}} .
$$

After calculating the transmission coefficient, the electronic conductance $G(E)$ is easily obtained from the Landauer-Buttiker formalism [35]:

$$
G(E)=\frac{4 e^{2}}{h} \sum_{m=0}^{p-1} T_{m},
$$

where $p$ represents the number of carbon atoms, and the factor of 4 accounts for both the spin and valley degeneracy. The physical parameters used in this paper are $L_{b}=L_{w}=1.2 \mathrm{~nm}, v_{F}=$ 
$1 \times 10^{6} \mathrm{~m} \cdot \mathrm{s}^{-1}$, and $\hbar=1.0545718 \times 10^{-34} \mathrm{~m}^{2} \cdot \mathrm{kg} \cdot \mathrm{s}^{-1}$. The number of carbon atoms, barrier velocity, and applied voltage are taken as parameters of the simulation.

\section{Results and discussion}

In Fig. 2a - 2c, we plot the variation of the transmission coefficients as a function of the incident energy for three different barrier velocities. We also considered different widths of the nanoribbon by varying the number of carbon atoms between 7 and 13. The applied voltage is switched off, and we limit our interest to the first $m=1$ mode. When the barrier velocity is equal to the Fermi velocity $\left(v_{b}=v_{F}\right.$ ) (Fig. $2 \mathrm{a}$ ), the transmission coefficient is equal to unity, and the nanoribbon appears transparent to the electrons regardless of their energies. In other words, in the absence of defects, the nanoribbon can be fully transparent, which can be used for the realization of on/off states in graphene-based transistors. Furthermore, it is important to note that the transmission coefficient starts from lower energies when the number of carbon atoms is high. For instance, the transmission starts at $E=2.1 \mathrm{eV}$ for $p=7$ but begins at $E=1.1 \mathrm{eV}$ for $p=13$. In Fig. $2 \mathrm{~b}$, the barrier velocity is fixed at $v_{b}=1.5 v_{F}$, and the transmission coefficient exhibits an oscillatory behavior where the starting energy value (threshold energy) of the transmission coefficient is inversely related to the number of carbon atoms. The transmission coefficient peaks become wider and tend to unity for higher-incident electron energies. To get a consistent comparison, Fig. 2c plots the transmission coefficient for a barrier velocity less than the Fermi velocity $\left(v_{b}=0.5 v_{F}\right)$. From this figure, the transmission coefficient presents additional oscillations compared to the $v_{b}=1.5 v_{F}$ case, and the peaks are sharper compared to previous cases. All of these findings show that more resonant states can be obtained by inserting a substrate on top of the nanoribbon to ensure that its barrier velocity is less than the Fermi velocity $\left(v_{b}<v_{F}\right)$. In general,

modulating the velocity in the barrier regions is crucial for obtaining the desired transmission and conductance.

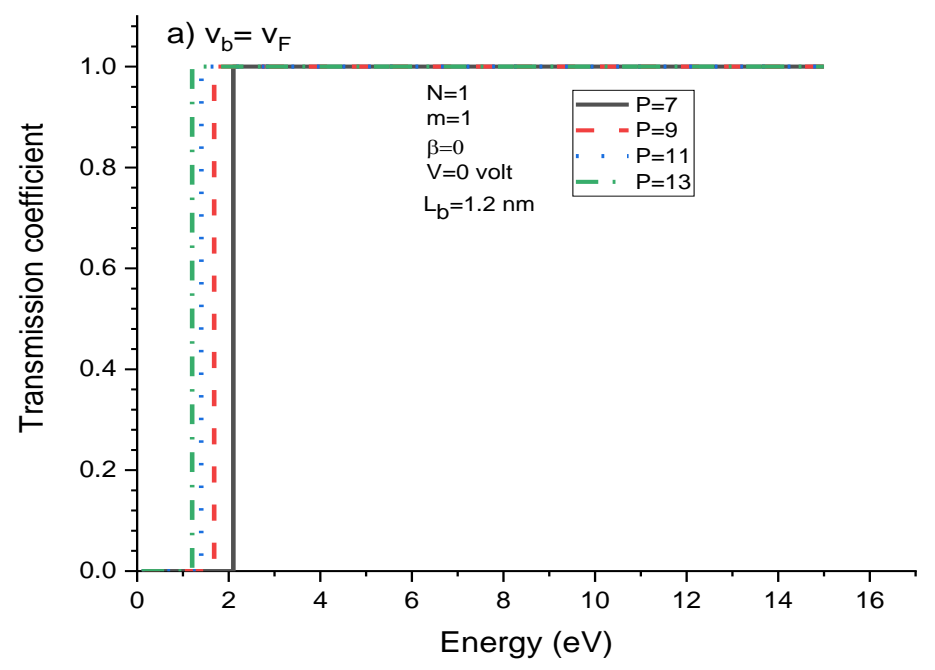



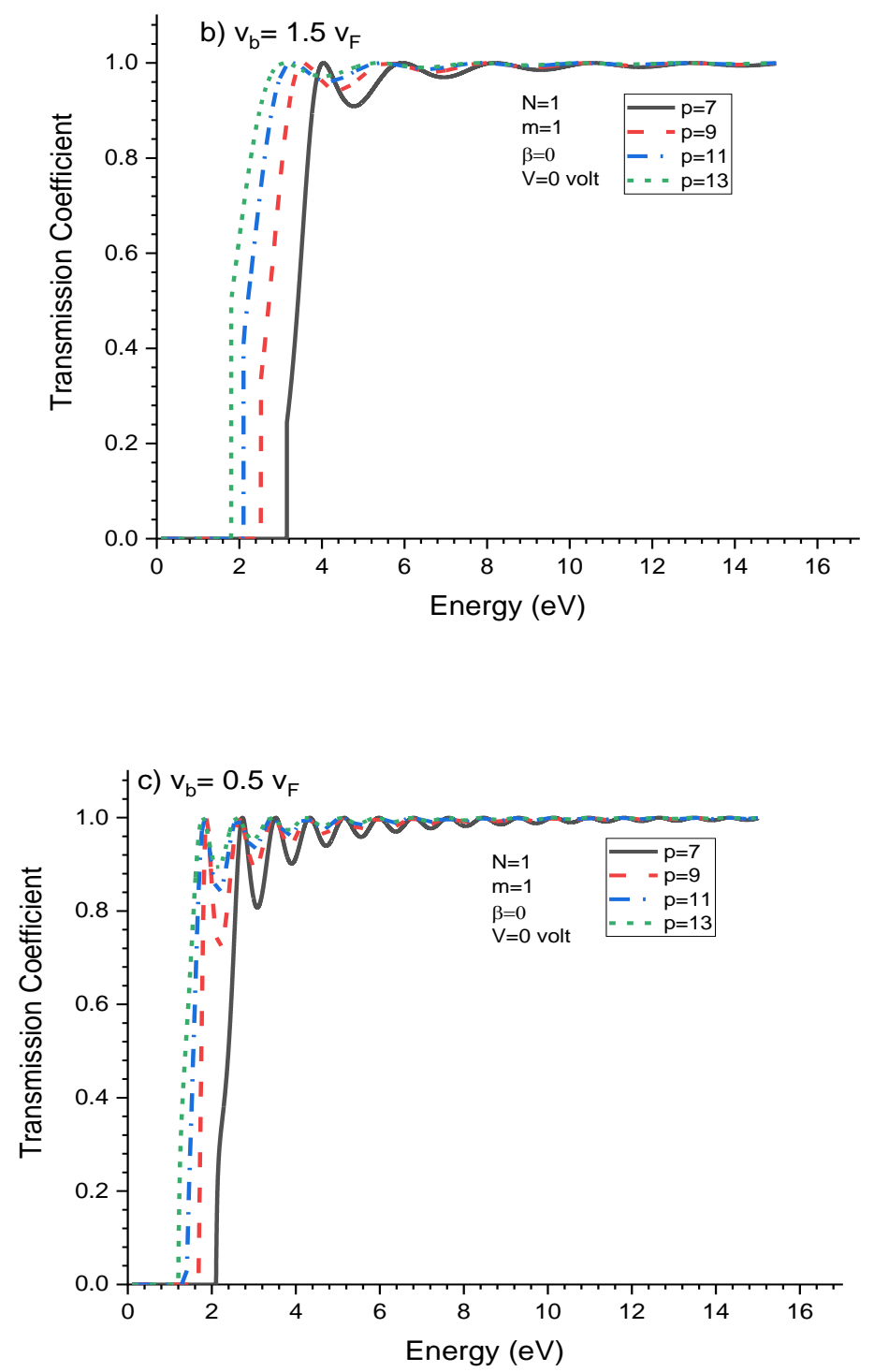

Figures 2: Transmission coefficient as a function of incident energy for different barrier velocities a) $v_{b}=v_{F}, \mathrm{~b}$ ) $v_{b}=1.5 v_{F}$, and c) $v_{b}=0.5 v_{F}$.

Figs. 3a-3c plot the transmission coefficient as a function of the incident energy for three different modes of propagation ( $m=1,3$, and 5). In this case, we fix the number of carbon atoms at $p=7$, the barrier velocity is set to $v_{b}=0.5 v_{F}$, and the voltage is switched off. By examining these figures, we observe that the transmission coefficient exhibits oscillations with increasing energy, and the number of peaks diminishes for higher modes. For instance, only 6 peaks are observed for $m=5$; however, 11 peaks emerge for $m=3$. In addition, we observe that the threshold energy (starting energy) for the transmission coefficient increases with $m$. For example, the transmission coefficient starts at $2 \mathrm{eV}$ for $m=1$ but emerges at $10.5 \mathrm{eV}$ for $m=5$. Furthermore, we 
observe that for $E>10 \mathrm{eV}$, the transmission coefficients exhibit oscillations for $m=3$ and 5, but for $m=1$ (see Fig. 3a), the transmission coefficient reaches unity and plateaus for $E>10 \mathrm{eV}$. The oscillations observed in Figs. $3 a-3 c$ can be interpreted as follows: when the Dirac electron energy is very close to the eigenenergy of the quantum wells formed between the barriers (modulated by the substrates), the electron wavefunction is trapped in the well regions, reflecting back and forth between the barriers in such a phase as to produce constructive interference. This interference is the origin of the resonant feature of the transmission coefficient.

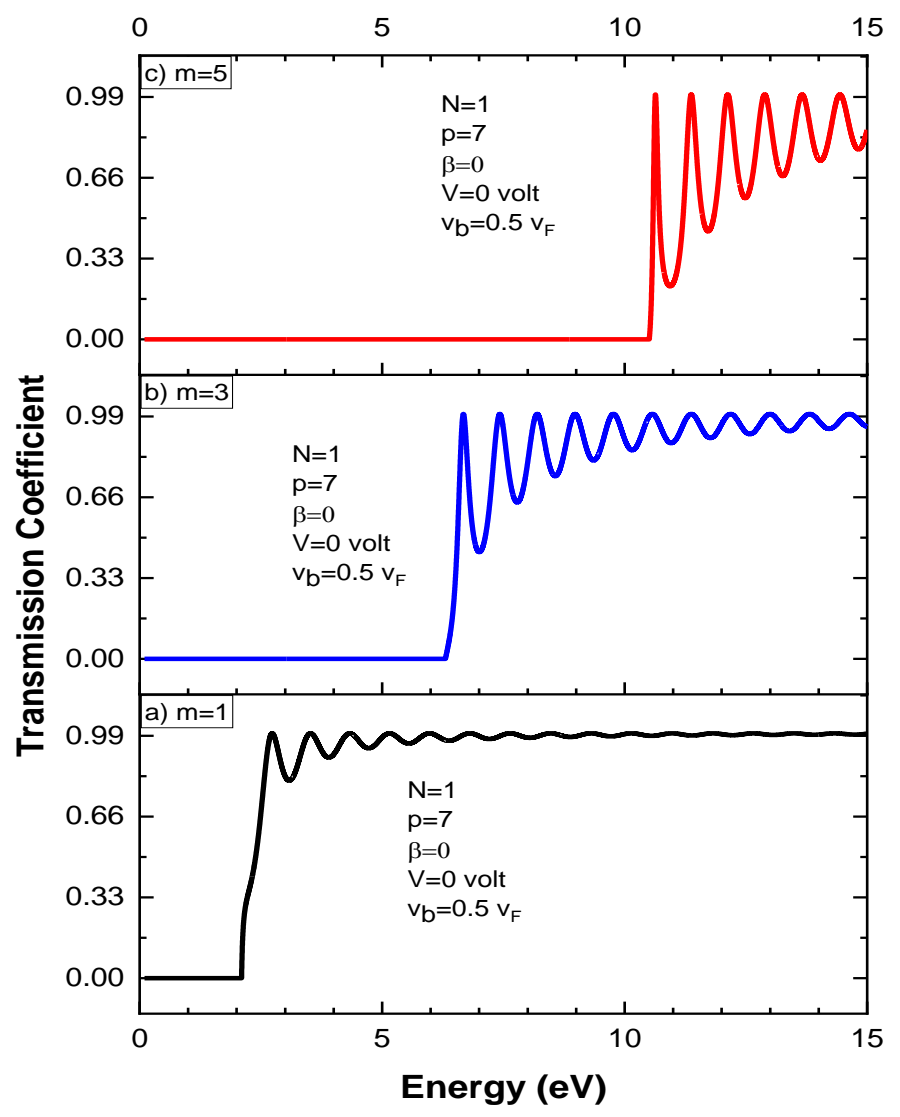

Figures 3: Transmission coefficient as a function of incident energy for three modes a) $m=1$, b) $m=3$, and c) $m=$ 5

Another important physical parameter is the applied voltage. Figs. $4 a-4 c$ plot the transmission coefficient as a function of the incident energy for three different applied voltages $(V$ $=0,5$, and 7 volts) for the $m=1$ mode. The heterostructure contains one barrier, and the number of carbon atoms is fixed to $p=7$. For these parameters, the threshold energy does not depend on the value of the applied voltage, with all the transmissions starting at $E=2 \mathrm{eV}$. In the absence of an applied voltage (Fig. 4a), the transmission coefficient exhibits more oscillations (compared to other cases when the external voltage is applied); however, their peaks are less sharp. When the applied voltage is switched on (Figs. $4 \mathrm{~b}$ and $4 \mathrm{c}$ ), the transmission coefficients exhibit sharper peaks 
than those obtained in Fig. 4a, and the amplitudes of the oscillations are large. Furthermore, we observe that the transmission coefficients vanish for certain energies centered around the value of the applied voltage potential, indicating that the electronic transmission is suppressed. For instance, when the applied voltage, $V$, is 5 volts, the transmission vanishes for all energies between 4 and 6 $\mathrm{eV}$; when $V=7$ volts, the transmission coefficient is zero for all incident energies between 6 and 8 $\mathrm{eV}$. In addition, we find that the transmission gap separates two series of oscillations. The first series is situated at the left of the gap and contains sharper peaks; however, the second series exhibits damped oscillations with small amplitudes. Moreover, we note that the number of resonant states increases with the applied voltage. For instance, three peaks emerge in the first series of resonant energies for $V=5$ volts (Fig. $4 \mathrm{~b}$ ), and five peaks in the first series of resonant energies emerge for $V=7$ volts (Fig. 4c). The overall behavior of these transmission coefficients provides guidance for nanoribbon applications since they enable the creation of on/off states that are required in the design of field-effect transistors. In other words, the on/off states can be obtained simply by modulating the incident energy of Dirac electrons through the armchair nanoribbon.

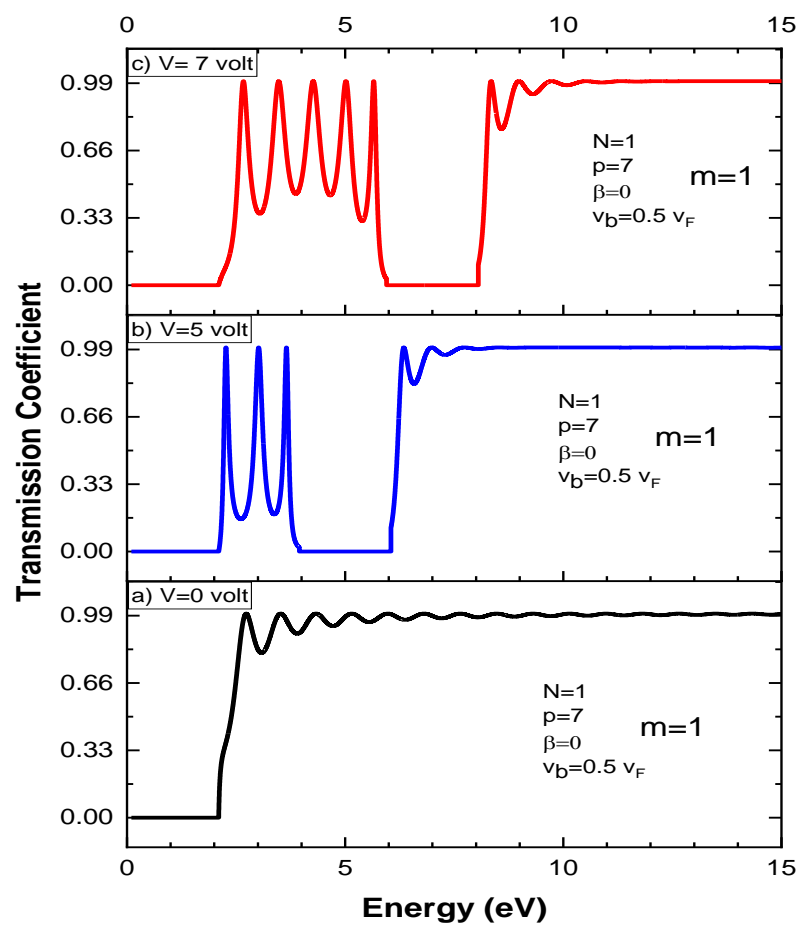

Figures 4: Transmission coefficient as a function of incident energy for three applied voltage a) $V=0$, b) $V=5$, and c) $V=7$ volts

We now investigate the effect of multiple barriers on the transmission coefficient and conductance. Such barriers can be realized by the deposition of additional substrates on top of the nanoribbon. In Fig. 5, we consider an AGN structure containing 7 carbon atoms without an applied voltage for the first mode $(m=1)$. The barrier velocity is fixed at $v_{b}=0.5 v_{F}$. We plot the transmission coefficient as a function of incident energy for three barrier numbers $(N=1,3$, and 
5). As shown in these figures, the number of peaks strongly increases, and their amplitudes increase with the number of barriers (the number of resonant states increases by adding more barriers on the top of the nanoribbon). Fig. 6 depicts the same structure in Figs. $5 \mathrm{a}-5 \mathrm{c}$ but with an external voltage of $V=5$ volts. The transmission coefficient presents the same oscillatory behavior, which contains a gap for all energies situated around the applied voltage value (5 volts). In this region, the evanescent character of the wavefunctions is responsible for reducing the transmission coefficient, and the Dirac electrons are consequently backscattered. To the right of the transmission gap, these peaks are separated by parabolic gaps for $N=1$ and 2 . However, for $N=5$, the peaks are longer than those in the cases for $N=1$ and 2 and are separated by straight gaps (Fig. 6c).

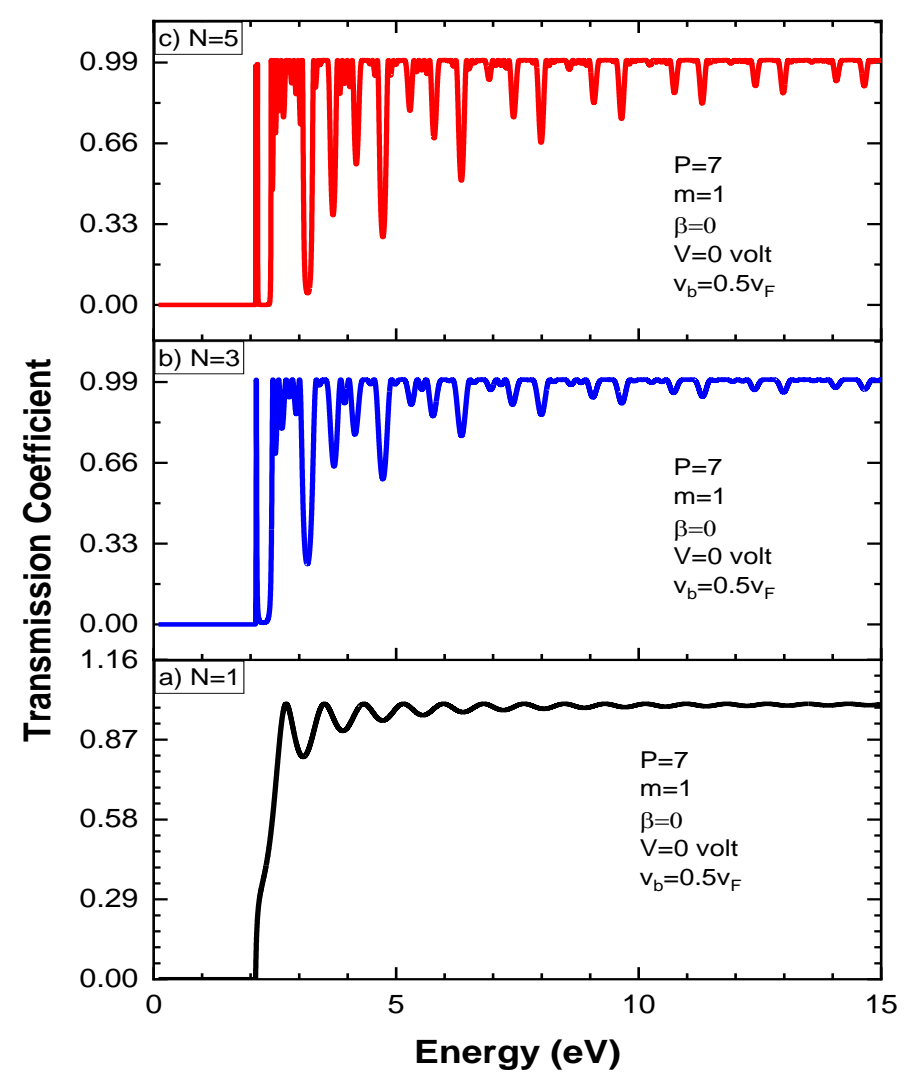

Figure 5: Transmission coefficient as a function of incident energy for three different barrier numbers a) $N=1$, b) $N=3$, and c) $N$ $=5$ 


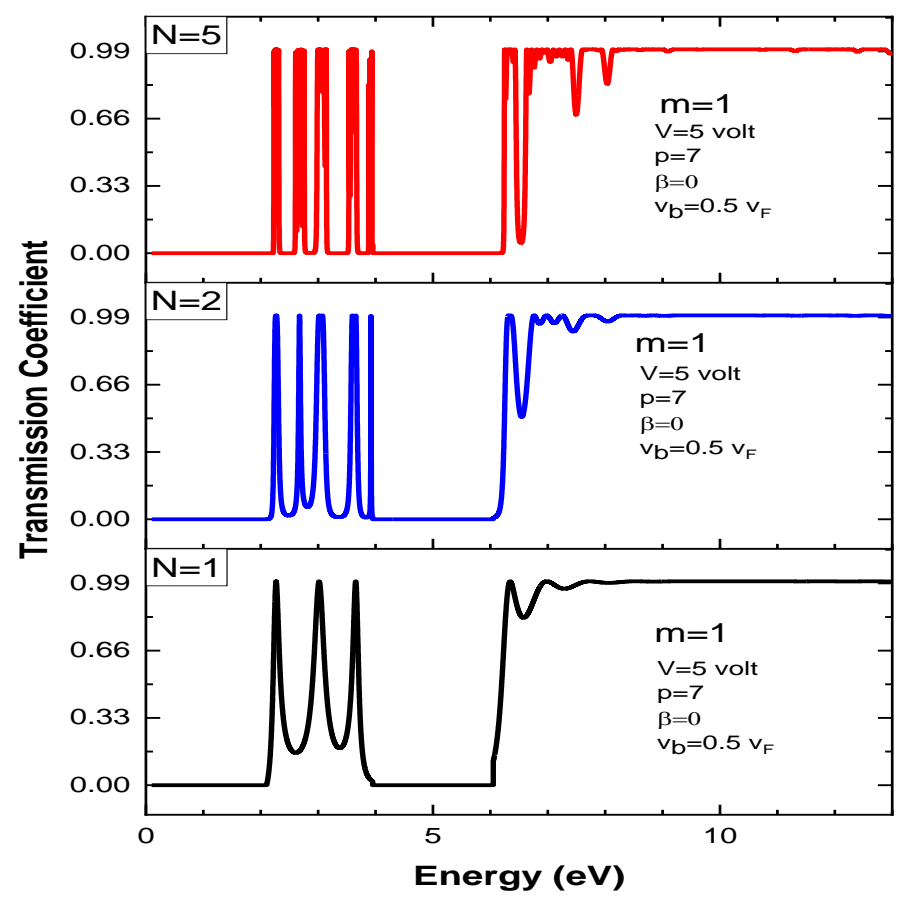

Figures 6: Transmission coefficient as a function of incident energy for three barrier numbers a) $N=1, \mathrm{~b}) N=3$, and c) $N=5$ for an applied voltage of 5 volts

In the remainder of this paper, we discuss the variations of the electronic conductance in AGNs restricted to one barrier. Figs. $7 \mathrm{a}-7 \mathrm{c}$ plot the variation of the conductance for three different barrier velocities $v_{b}=0.5 v_{F}, v_{F}$, and $1.5 v_{F}$ without an applied voltage. When $v_{b}=v_{F}$ (Fig. 7a), the electronic conductance shows a series of "jumps" when the incident energy is increased. These jumps appear when the energy increases, which correspond to new modes of propagation along the channel. Furthermore, for a given energy interval, the number of jumps increases with the number of carbon atoms. For $v_{b}=1.5 v_{F}$ (Fig. $7 \mathrm{~b}$ ), the electronic conductance peaks are curved since the transmission coefficients oscillate, as shown in Fig. 2b. In addition, the conductance starts at lower energies for a higher number of carbon atoms, but their steps are narrower. Fig. 7c plots the conductance for $v_{b}=0.5 v_{F}$ and shows that the number of jumps increases more than the previous case of $v_{b}=1.5 v_{F}$ with their shapes becoming rounder. Moreover, the threshold energies are shifted to lower values. Finally, at lower energies, the conductances are similar to each other regardless of the number of carbon atoms; however, we observe a large splitting at higher energies. 

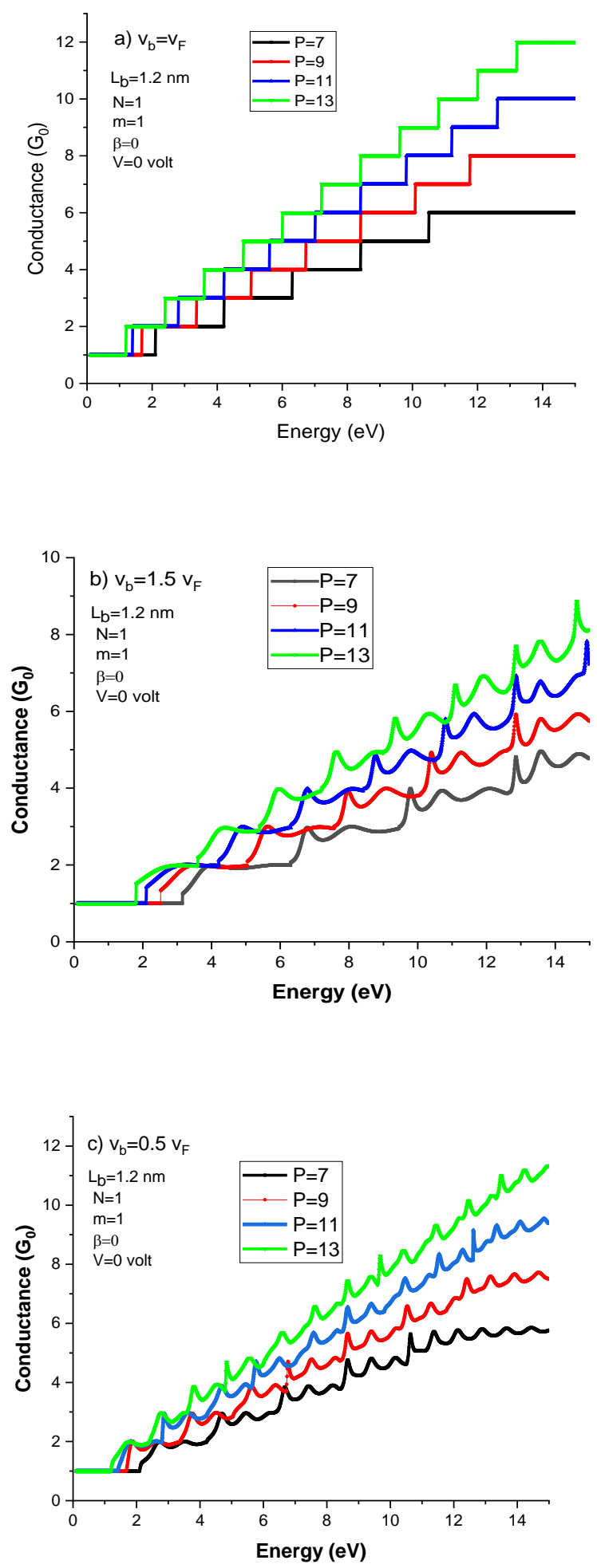

Figure 7: Conductance as a function of incident energy for different barrier velocities a) $v_{b}=v_{F}$, b) $v_{b}=1.5 v_{F}$, and c) $v_{b}=0.5 v_{F}$. 
Finally, Fig. 8 plots the electronic conductance as a function of the incident energy for three different applied voltages $(V=3,5$, and 7 volts) for the case of one barrier and a barrier velocity of $v_{b}=0.5 v_{F}$. We find that the number of jumps is identical regardless of the applied voltage on the barrier. The first jump exhibits a constant value with higher jumps exhibiting rounded oscillations. The number of oscillations increases with the voltage and incident energy. In addition, we find that the threshold energy moves to higher values when the applied voltage is increased. We also note that the threshold energy is equal to the applied voltage and demonstrates that an experiment can obtain the desired threshold energy by selecting the appropriate applied voltage. For higher energies, the peaks of the conductance are reduced and tend to the same value.

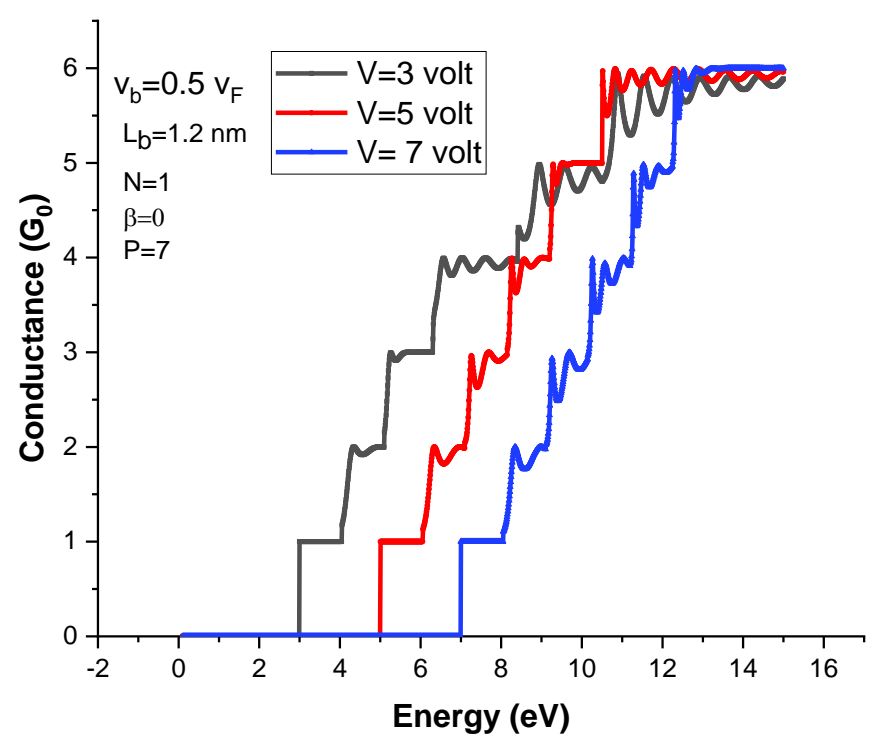

Figure 8: Conductance as a function of incident energy for different applied voltages

\section{Conclusion}

In this work, we have examined the transmission coefficient and electronic conductance across multibarrier armchair graphene nanoribbons. These barriers can be realized by depositing substrates on the AGN, which can also tune the barrier velocity $v_{b}$ and applied voltage. Our results indicate that the transmission coefficient is equal to unity for all energies when the Fermi and barrier velocities are equal $\left(v_{b}=v_{F}\right)$, and the AGN is transparent to electron transmission. However, for $v_{b} \neq v_{F}$, the transmission coefficient oscillates, and the peaks are sharper for $v_{b}<$ $v_{F}$ compared to the $v_{b}>v_{F}$ case. We also find that the threshold energy of the transmission coefficient increases for higher modes and a large number of atoms. In addition, the transmission coefficients exhibit gaps for certain external voltage values. These gaps are centered around the applied voltage and enable the realization of optoelectronic devices based on (on/off) states such as field-effect transistors. Furthermore, the electronic conductance of an AGM exhibits sudden 
"jumps" when the incident energy is increased. The jumps are "rounded" for $v_{b} \neq v_{F}$ but become more abrupt when $v_{b}=v_{F}$. Moreover, the conductance increases with the number of carbon atoms due to the additional modes. Finally, the effect of an applied voltage on the conductance was examined, and our findings indicate that the threshold energy of the conductance is shifted towards higher energies when the applied voltage is increased. Collectively, our results provide theoretical

guidance in the design and realization of other nanoribbon-based devices [38] for promising applications.

\section{Acknowledgments}

B.M.W. acknowledges financial support from the Office of Naval Research (Grant N00014-18-12740).

\section{References}

[1] C. Berger, Z.M. Song, X.B. Li, X.S. Wu, N. Brown, C. Naud, D. Nato, T.B. Li, J. Hass, A.N. Marchenkov, E.H. Conrad, P.N. First, W.A. de Heer, Science 312 (2006) 1191-1196.

[2] Ren-Bin Gao, Xiao-Fang Peng, Xiang-Tao Jiang, Shi-Hua Tan, Meng-Qiu Long. Organic Electronics 67 (2019) 57-63.

[3] Ren-Bin Gao, Xiao-Fang Peng, Ke-Qiu Chen. Physica E 104 (2018) 302-308

[4] Xiao-Fang Peng, Ke-Qiu Chen, Xin-Jun Wang, Shi-Hua Tan. Carbon 100 (2016) 36-41

[5] A. H. Castro Neto, F. Guinea, N.M.R. Peres, K.S. Novoselov, A.K. Geim, Reviews of Modern Physics 81 (2009) 109-162.

[6] L. Brey, H. Fertig, Electronic states of graphene nanoribbons studied with the Dirac equation, Phys. Rev. B 73 (23) (2006) 235411.

[7] H. Guo, N. Lu, J. Dai, X. Wu, X.C. Zeng, Phosphorene nanoribbons, phosphorus nanotubes, and van der Waals multilayers, J. Phys. Chem. C 118 (2014) 14051.

[8] R. Sako, H. Hosokawa, H. Tsuchiya, Computational study of edge configuration and quantum confinement effects on graphene nanoribbon transport, IEEE Electron Device Lett. 32 (Jan 2011) 6-8.

[9] Y. Lu, S. Zhao, Y. Zhang, H. Liu, W. Lu, W. Liang, Valley-polarized insulating states in zigzag silicene nanoribbons, Mater. Res. Express 1 (2014) 045009. 14051-14059. 
[10] R. Tsu, L. Esaki, Tunneling in a finite superlattice, Appl. Phys. Lett. 22 (1973) 562-564.

[11] C. Bai, X. Zhang, Klein paradox and resonant tunneling in a graphene superlattice, Phys. Rev. B 76 (Aug 2007) 075430.

[12] H. Li, J.M. Shao, H.B. Zhang, G.W. Yang, Electrical tuning of transport properties of topological insulator ultrathin films, Nanoscale 6 (2014) 3127-3137.

[13] M. Barbier, F.M. Peeters, P. Vasilopoulos, J.M. Pereira, Dirac and Klein-Gordon particles in one-dimensional periodic potentials, Phys. Rev. B 77 (2008) 115446.

[14] C.-H. Park, L. Yang, Y.-W. Son, M.L. Cohen, S.G. Louie, New generation of massless Dirac fermions in graphene under external periodic potentials, Phys. Rev. Lett. 101 (2008) 126804.

[15] M. Barbier, P. Vasilopoulos, F.M. Peeters, Dirac electrons in a kronig-penney potential: dispersion relation and transmission periodic in the strength of the barriers, Phys. Rev. B 80 (2009) 205415.

[16] N. Abedpour, A. Esmailpour, R. Asgari, M. R. R. Tabar, Conductance of a disordered graphene superlattice, Phys. Rev. B 79 (2009) 165412.

[17] H. Cheraghchi, A. H. Irani, S.M. Fazeli, R. Asgari, Metallic phase of disordered graphene superlattices with long-range correlations, Phys. Rev. B 83 (2011) 235430.

[18] A. Esmailpour, H. Meshkin, R. Asgari, Conductance of graphene superlattices with correlated disorder in velocity profiles, Solid State Commun. 152 (20) (2012) 1896-1901

[19] R. P. Tiwari, D. Stroud, Tunable band gap in graphene with a noncentrosymmetric superlattice potential, Phys. Rev. B 79 (2009) 205435.

[20] L.-G. Wang, S.-Y. Zhu, Electronic band gaps and transport properties in graphene superlattices with one-dimensional periodic potentials of square barriers, Phys. Rev. B 81 (2010) 205444.

[21] M. Barbier, P. Vasilopoulos, F.M. Peeters, Extra Dirac points in the energy spectrum for superlattices on single-layer graphene, Phys. Rev. B 81 (2010) 075438.

[22] J. R. F. Lima, Electronic structure of a graphene superlattice with massive Dirac fermions, J. Appl. Phys. 117 (8) (2015) 084303. 
[23] F. Zhai, K. Chang, Theory of huge tunneling magnetoresistance in graphene, Phys. Rev. B 77 (2008) 113409.

[24] M. Ramezani Masir, P. Vasilopoulos, A. Matulis, F.M. Peeters, Direction dependent tunneling through nanostructured magnetic barriers in graphene, Phys. Rev. B 77 (2008) 235443.

[25] M. Ramezani Masir, P. Vasilopoulos, F.M. Peeters, Tunneling, conductance, and wavevector filtering through magnetic barriers in bilayer graphene, Phys. Rev. B 79 (2009) 035409.

[26] L. Dell'Anna, A. De Martino, Multiple magnetic barriers in graphene, Phys. Rev. B 79 (2009) 045420 .

[27] P. M. Krstaji'c, P. Vasilopoulos, Ballistic transport through graphene nanostructures of velocity and potential barriers, J. Phys. Condens. Matter 23 (2011) 135302.

[28] F. Zhai, K. Chang, Valley filtering in graphene with a Dirac gap, Phys. Rev. B 85 (2012) 155415.

[29] D. Moldovan, M. Ramezani Masir, L. Covaci, F. M. Peeters. Resonant valley filtering of massive Dirac electrons, Phys. Rev. B 86 (2012) 115431.

[30] Yang Lu, Jing Guo, Nano Research 3 (2010) 189.

[31] J. R. Lima, Controlling the energy gap of graphene by Fermi velocity engineering, Phys. Lett. A 379 (2015) 179-182.

[32] A. Diaz-Fernandez, L. Chico, J.W. Gonzalez, F. Dominguez-Adame, Tuning the Fermi velocity in Dirac materials with an electric field, Sci. Rep. 7 (2017) 8058.

[33] Haiyan Wang, Chao Wu, Fang Xie, Xiaojiao Zhang, Guanghui Zhou. Physica B 533. (2018) 40.

[34] Benliang Zhou, Benhu Zhou, Guanghui Zhou. Electronic tunneling through a potential barrier on the surface of a topological insulator. Modern Phys. Lett. B. 30 (2016) 1650416

[35] A.C.S. Nascimento, R. P. A. Lima, M. L. Lyra, J. R. F. Lima. Electronic transport on graphene armchair-edge nanoribbons with Fermi velocity and potential barriers. Physics Letters A 383 (2019) 2416-2423.

[36] B. Zhou, B. Zhou, W. Liao, G. Zhou, Electronic transport for armchair graphene nanoribbons with a potential barrier, Phys. Lett. A 374 (5) (2010) 761-764

[37] J. Tworzydło, B. Trauzettel, M. Titov, A. Rycerz, C.W. Beenakker, Sub-Poissonian shot noise in graphene, Phys. Rev. Lett. 96 (2006) 246802

[38] B. M. Wong, S. H. Ye, G. O’Bryan, Nanoscale, 4 (2012) 1321-1327 
[39] H. Dakhlaoui, W. Belhadj, B. M. Wong, Results in Physics 16 (2021) 104403 